\title{
Drug Accountability Treatment Return Date
}

National Cancer Institute

\section{Source}

National Cancer Institute. Drug Accountability Treatment Return Date. NCI Thesaurus.

Code C83167.

The The drug accountability information that indicates the date that the study treatment was returned. 